\title{
Antidepressants are not safe during pregnancy and in women of child bearing age.
}

\author{
Alain Braillon ${ }^{1}$, Susan Bewley ${ }^{2}$, Aubrey Blumsohn ${ }^{1}$, florian naudet ${ }^{1}$, Jean-Louis \\ Montastruc $^{3}$, and Joel Lexchin ${ }^{4}$ \\ ${ }^{1}$ Affiliation not available \\ ${ }^{2}$ Women's Health Academic Centre, King's College \\ ${ }^{3}$ Toulouse University Hospital \\ ${ }^{4}$ York University
}

June 30, 2021

Antidepressants are not safe during pregnancy and in women of child bearing age.

Alain Braillon (1), Susan Bewley(2), Aubrey Blumsohn(3), Florian Naudet (4) Jean-Louis Montastruc(5), Joel R Lexchin(6)

(1) Previously senior consultant, Amiens, France braillon.alain@gmail.com

(2) Department of Women \& Children's Health, King's College London, London, UK

(3) Previously Senior Lecturer in Medicine, University of Sheffield, Sheffield, UK

(4) University of Rennes 1, Rennes, France Clinical Investigation Center (INSERM 1414) and Adult Psychiatry Department, Rennes University Hospital, Rennes, France

(5) Medical and Clinical Pharmacology, University Hospital-Faculty of Medicine, Toulouse, France

(6) School of Health Policy and Management Faculty of Health, York University, Toronto, Ontario, Canada

Correspondance

Alain Braillon, 27 rue Voiture, 80000 Amiens, France. braillon.alain@gmail.com

$757 \mathrm{w}+9$ ref. (Limit 800 words +10 references)

Keywords: risk minimization measures; drug safety; serotonin reuptake inhibitors; teratogenicity; developmental disorders; post-partum hemorrhage; EBM

No funding

CoI: Alain Braillon, JL and SB are among industry independent experts on Jeanne Lenzer's list (https://jeannelenzer.com/list-independent-experts). SB chairs Healthwatch, a charity promoting science and integrity in healthcare (https://www.healthwatch-uk.org/) and her interests are declared at https://www.whopaysthisdoctor.org/doctor/58/active. JL received payments for writing a brief in an action for side effects of a drug for Michael F. Smith, Lawyer and a second brief on the role of promotion in generating prescriptions for Goodmans LLP in 2017-2020. He is a member of the Foundation Board of Health Action International. He receives royalties from University of Toronto Press and James Lorimer \& Co. Ltd. for books he has written. 
Cabaillot and colleagues' pharmaco-epidemiological study of antidepressants use during pregnancy in France raises a number of questions about the validity of their conclusions.(1)

Firstly, what is their basis for making the claim that "[selective serotonin-norepinephrine reuptake inhibitors] appear to be safe in pregnant women"?(1) This claim ignores significant evidence to the contrary:

a) the odds ratio for maternal use of serotonin reuptake inhibitor antidepressants (SRIs) (we use SRI to refer to both serotonin-norepinephrine reuptake inhibitors and so-called 'selective' serotonin reuptake inhibitors as selectivity is restricted to the experimental setting) during the first trimester of pregnancy and the presence of congenital heart defects is 1.57 (95\% CI 1.25-1.97) for paroxetine, 1.36 (95\% CI 1.08-1.72) for fluoxetine and 1.29 (95\% CI 1.14-1.45) for sertraline.(2) Further, observational studies reported developmental disorders that are in line with experimental studies and with human infant MRI studies: SRIs exposure has an association with changes in brain structure, white matter microstructure, brain connectivity, and cerebral metabolism, particularly in brain regions critical to emotional processing;(3)

b) previous warnings about SRIs' harms in core clinical journals were overlooked; $(4,5)$

c) post-partum hemorrhage, a serious SRI complication acknowledged by Cabaillot and colleagues, is a very serious issue; with cardiovascular conditions it is the most common underlying causes of pregnancy-related death.(6)

Secondly, Cabaillot et al misrepresent the references that they cite in their statement that "[selective serotonin-norepinephrine reuptake inhibitors] are recommended by the National Agency for Health and Medicines (ANSM) and the 2018 International Cochrane Review International Publications "?(1)

a) the link for the recommendation (ref. 19 in Cabaillot et al) does not work and the summary of product characteristics for fluoxetine warns "Overall, the data suggest that the risk of cardiovascular malformation in children after maternal exposure to fluoxetine is approximately $2 / 100 \ldots$ Fluoxetine should not be used during pregnancy unless the clinical condition of the patient requires treatment with fluoxetine and justifies the potential risk to the fetus".(7)

b) the Cochrane Review (ref. 20 in Cabaillot et al) is about postnatal depression, not pregnancy. It concluded: "Due to the limitations of the current evidence base, such as the low statistical power of the included studies, it is not possible to draw any clear conclusions about the effectiveness of antidepressants for the prevention of postnatal depression."

Thirdly, Cabaillot and colleagues should provide data (malformations, deprescription) according to the various classes of antidepressants and their princeps. The risk of malformations varies among antidepressant classes and within classes, e.g., it is highest for SRIs containing paroxetine.(2)

Fourthly, the widespread prescribing of antidepressants in France may be the result of France's universal healthcare scheme consistently refusing to reimburse effective psychotherapy. In contrast is the 2008 Improving Access to Psychological Therapies strategy in England that provides free and direct access to psychotherapy without barriers.(8)

Overplaying benefits and downplaying harms are not serving the patient's interests. All prescribers must be warned that antidepressants have not been shown to be risk free during pregnancy, either for the woman or her fetus. Quantitative explanation of the degree of risk expressed in easily understood terms such as number needed to harm must be provided, not binary assertions of apparent "safety" based on a value judgment only women should make. The well documented harms of SRIs during pregnancy contrast with purported benefits _a limited effectiveness of SRIs in the general population and no controlled trials during pregnancy. Available SRI vs placebo trials are short-term (6-8 weeks) and although the decreases in depression scores are statistically significant, their clinical relevance is doubtful.(9) In contrast, many patients with depression respond to judicious 'watchful waiting' and psychotherapies successfully treat most episodes of even persistent depression. These interventions are evidence-based in real-life settings: their benefits persist over the long term (improving self-esteem, agency, and social functioning and, they are safe.(9) Evidence based 
psychosocial interventions must be the preferred initial option in treating depression, especially in women of child bearing age as many pregnancies are unintended.(4)

Regulatory agencies must implement and monitor a series of risk minimization measures for SRIs as for other teratogens: a) an assessment of each woman's potential to become pregnant; b) shared decision making about the risks of antidepressant treatment in general and specifically during pregnancy, and an explanation about the need for effective contraception throughout treatment; c) informed pregnancy testing before and during treatment as needed; d) a 'risk acknowledgement form', in which the woman and prescriber confirm that appropriate counselling has been given and understood. Finally, prescription of antidepressants during pregnancy should be recorded in specific registries to closely monitor clinical outcomes.

References

1. Cabaillot A, Bourset A, Mulliez A et al. Trajectories of antidepressant drugs during pregnancy: A cohort study from a community-based sample. Br J Clin Pharmacol. 2021;87(3):965-987.

doi: $10.1111 /$ bcp.14449.

2. De Vries C, Gadzhanova S, Sykes MJ, Ward M, Roughead E. A Systematic Review and Meta-Analysis Considering the Risk for Congenital Heart Defects of Antidepressant Classes and Individual Antidepressants. Drug Saf. 2020;44(3):291-312.

doi: $10.1007 / \mathrm{s} 40264-020-01027-\mathrm{x}$

3. Lugo-Candelas C, Cha J, Hong S, et al. Associations Between Brain Structure and Connectivity in Infants and Exposure to Selective Serotonin Reuptake Inhibitors During Pregnancy. JAMA Pediatr. 2018; 172(6):525-533. doi: 10.1001/jamapediatrics.2017.5227.

4. Braillon A, Bewley S. Prescribing in pregnancy shows the weaknesses in pharmacovigilance. BMJ. 2018;361:k2334. doi: 10.1136/bmj.k2334.

5. Braillon A, Bewley S. The easy misuse of antidepressants during pregnancy is depressing. Br J Clin Pharmacol. 2018;84(10):2445-2446. doi: 10.1111/bcp.13713.

6. Davis NL, MPH, Smoots AN, Goodman, DA. Pregnancy-Related Deaths: Data from 14 U.S. Maternal Mortality Review Committees, 2008-2017. Maternal Mortality Review Data-Brief. 2019. Available at https://www.cdc.gov/reproductivehealth/maternal-mortality/erase-mm/MMR-DataBrief_2019-h.pdf Accessed 27 May 2020.

7. French National Agency for Health and Medicines (ANSM). Fluoxetine: Summary of Product Characteristics. 3 April 2016. Available at http://agence-prd.ansm.sante.fr/php/ecodex/rcp/R0278605.htm Accessed 27 May 2021.

8. Thornicroft G. Improving access to psychological therapies in England. Lancet. 2018;391(10121):636-637. doi: 10.1016/S0140-6736(17)32158-X.

9. Braillon A, Lexchin J, Noble JH, Menkes D, M'sahli L, Fierlbeck K, Blumsohn A, Naudet F. Challenging the promotion of antidepressants for nonsevere depression. Acta Psychiatr Scand. 2019;139(3):294-295. doi: $10.1111 /$ acps. 13010. 